\title{
A new southern high-latitude index
}

\author{
P. Ballatore ${ }^{1}$, C. G. Maclennan ${ }^{2}$, M. J. Engebretson ${ }^{3}$, M. Candidi ${ }^{1}$, J. Bitterly ${ }^{4}$, C.-I. Meng ${ }^{5}$ and G. Burns ${ }^{6}$ \\ ${ }^{1}$ IFSI/CNR, c.p.27, 00044 Frascati, Rome, Italy \\ 2 Bell Laboratories, Lucent Technologies, Murray Hill, NJ 07974 USA \\ ${ }^{3}$ Dept. of Physics, Augsburg College, Minneapolis, MN 55454 USA \\ ${ }^{4}$ Ecole et Observatoire de Physique du Globe, Strasbourg, France \\ 5 Johns Hopkins Applied Physics Laboratory, Laurel, MD 20723 USA \\ ${ }^{6}$ Australian Antarctic Division, Hobart, Australia
}

Received: 4 July 1997 / Revised: 24 January 1998 / Accepted: 12 May 1998

\begin{abstract}
We have developed and examined a new regional geomagnetic index AES-80, defined similarly to the classical auroral electrojet AE index, using data from five Antarctic stations located at corrected geomagnetic latitudes about $80^{\circ} \mathrm{S}$. Because only sparse ground-based information can be derived from auroral latitudes in the Southern Hemisphere, and because no index comparable to $\mathrm{AE}$ can be constructed from locations in the south, the possibility of using AES- 80 as a measure of high latitudes and polar cap activity is investigated. As a global average activity level indicator, it is found that in general AES-80 gives results rather similar to the classical AE index. However AES-80 provides a more robust measure of the occurrence of high-latitude geomagnetic activity.
\end{abstract}

Key words. Magnetospheric physics (auroral phenomena; polar cap phenomena).

\section{Introduction}

Two indices, the auroral electrojet (AE) and the polar cap (PC), have been developed in order to monitor geomagnetic activity at auroral and polar cap latitudes, respectively. The AE index (Davis and Sugiura, 1966) indicates the magnitude of auroral electrojet currents and, in particular, the occurrence of substorms (Baumjohann, 1986). The PC index provides information on the polar cap convection; therefore, it is regarded as a ground signature of interplanetary parameters, in particular of the IMF-Bz component (Troshichev et al., 1988). Some relationship between these two indices is

Correspondence to: P. Ballatore expected, at least because the field-aligned currents at the poleward auroral rim both influence geomagnetic perturbations at polar latitudes and feed the ionospheric auroral electrojets. A good correlation between the PC and $\mathrm{AE}$ indices has, in fact, been reported by $\mathrm{Ve}-$ nnerstrøm et al. (1991).

The Antarctic land mass does not permit a reasonable spacing of geomagnetic observatories around the nominal austral auroral oval, so the Southern Hemisphere equivalent of the Northern Hemisphere AE index cannot be constructed. However, reasonably good average auroral conjugacy, such as shown many years ago by aircraft flights in opposite hemispheres (e.g. Belon et al., 1969), suggests the northern and southern $\mathrm{AE}$ indices should be similar and that hemispheric differences, should they exist, would occur from hemispheric and/or seasonal asymmetries in ionospheric conductivities.

The Antarctic land mass provides the only opportunity for establishing an array of azimuthally spaced stations in the polar cap, in either hemisphere. Such an array would provide data to both complement and supplement the present use of single station observations at Vostok and Thule. Data from this array, at about $80^{\circ} \mathrm{S}$ geomagnetic latitudes, would provide good coverage of polar cap and interplanetary medium. Constructing an AE-type index from stations in this array can provide new information on the physical state of the region of magnetosphere (the deep magnetotail) that maps into the polar cap. Data from some of the initial high-latitude stations in the Antarctic have shown that substorm-like processes are unexpectedly present in the polar cap (Weatherwax et al., 1997).

The AE index is calculated as the difference between the upper (AU) and the lower (AL) envelope of magnetograms from 12 observatories located at geomagnetic latitudes between $60^{\circ}$ and $70^{\circ}$ and rather uniformly distributed over all longitudes. Maclennan et al. (1991) calculated a "southern AE index" using stations spaced all over the Antarctic continent, not just in the nominal auroral zone. They compared it 
with the northern AE. The good correlation found was due mostly to data in time interval (00-11) UT and this was attributed to the fact that almost all Antarctic stations are at corrected geomagnetic longitude between $0^{\circ} \mathrm{E}$ and $90^{\circ} \mathrm{E}$. The smaller southern values (compared to northern values, as shown by linear regression) were attributed to the different ionospheric conductivities during local summer and winter in the two hemispheres; in fact the period considered in the Maclennan et al. (1991) study, seven days in June 1982, is all in the austral winter season. The PC index nominally overcomes these seasonal differences as it is computed separately for the north and the south using one station in each hemisphere (Troshichev et al., 1988).

In the case of a contracted (poleward) auroral oval, the use of a PC index is not optimum. Although a good correlation between $\mathrm{PC}$ and $\mathrm{AE}$ was found by Vennerstrøm et al. (1991), they also noted that, when the auroral oval is shifted poleward, substorms occurring in those high latitudes were too weak and localized to be detected by the PC index.

In the present study we investigate a proposed new regional index, denoted AES-80, calculated like AE but using a chain of magnetometers at corrected geomagnetic latitudes near $80^{\circ} \mathrm{S}$.

\section{Data analysis and experimental observations}

To calculate the AES- 80 parameter we use the geomagnetic north-south $(H)$ components at one minute resolution from the five Antarctic stations listed in Table 1, which are all located at corrected geomagnetic latitude $\sim 80^{\circ} \mathrm{S}$. The time period analyzed covers May and June 1994.

The magnetometers located at McMurdo and at the two AGO sites are all three-axis fluxgate instruments (Engebretson et al., 1997). These are not observatorystandard magnetometers, but calibrations and engineering parameters are monitored from each instrument to ensure that the data are of good quality. The instruments are each oriented in a left-handed magnetic frame of reference (looking down on the Earth in Antarctica) with an increase in the geomagnetic south-north $(\mathrm{H}$ component) indicating an increase in the field in the northward direction, an increase in the geomagnetic west-east ( $D$-component) indicating an increase in the field in the eastward direction, and an increase in the vertical ( $Z$-component) indicating an increase in the field in the upward direction.
The instruments at Casey (Rycroft, 1982) and Dumont d'Urville (full information can be found at http://eopg.u-strasbg.fr/obsmag/observ.html) are also three-axis fluxgate instruments.

In agreement with the original definition of Davis and Sugiura (1966), we use the north-south geomagnetic component of the horizontal geomagnetic field. For purposes of comparison we note that the northern $\mathrm{AE}$ index uses the total horizontal component (e.g. WDCC2 for geomagnetism, 1993). This could introduce a systematic difference between the North and South results. In particular, the effect of field-aligned currents (FACs) is to skew the magnetic field from its average direction, causing the $D$-component to increase. Therefore indices involving the total geomagnetic horizontal component can attain, via the $D$-component, a considerable influence from the FACs; this effect should be even stronger at higher latitudes.

To study how this difference in calculation might affect the overall scale of AES-80, we have calculated the average variation of the total $H$ and of the north-south $H$ with respect to their average value. For example, during May 1994, these numbers are respectively 0.22 $\mathrm{nT}$ and $0.23 \mathrm{nT}$ at Casey (CSY); they are respectively $0.06 \mathrm{nT}$ and $-0.18 \mathrm{nT}$ at Dumont d'Urville (DRV). Therefore we see that the variations of total $H$ (used for AE calculation) are, on average, roughly equivalent to the variations of the north-south $H$. In the specific case of May at DRV, the absolute value of the variations of the north-south $H$ (used for AES-80) are greater than variations in the total $H$ (used for $\mathrm{AE}$ ). Thus, this difference in calculation cannot account for the different dynamic scales observed between the two indices; e.g. in Fig. 2, where AES- 80 is smaller than AE by a factor of about 3 .

The average value of $H$ has been calculated for each station for the two quietest days of each month and then subtracted from the data. As expected for polar latitudes, the quietest days do not always correspond to those identified by the $\mathrm{Kp}$ index. Therefore, our quietest days were found using a simple automatic routine whose results, in agreement with the classical visual inspection of magnetograms, are found to monitor the quietest days at single polar stations better than Kp (Ballatore et al., 1997). For the classical AE index as computed by WDC-C2 for geomagnetism, the average value that is subtracted from the data is calculated as the mean value over each whole month. Davis and Sugiura (1966) specified, however, that the quietest days should be taken into account for the background $H$ level subtraction. In particular, for our index, whose values
Table 1. Coordinates of stations used to calculate AES-80

\begin{tabular}{lllllc}
\hline Station name & Code & $\begin{array}{l}\text { Geographical } \\
\text { latitude }\end{array}$ & $\begin{array}{l}\text { Geographical } \\
\text { longitude }\end{array}$ & $\begin{array}{l}\text { Corrected } \\
\text { geomagnetic } \\
\text { latitude }\end{array}$ & $\begin{array}{l}\text { Corrected } \\
\text { geomagnetic } \\
\text { longitude }\end{array}$ \\
\hline AGO P1 & AP1 & $83.86^{\circ} \mathrm{S}$ & $129.61^{\circ} \mathrm{E}$ & $80.14^{\circ} \mathrm{S}$ & $16.75^{\circ} \mathrm{E}$ \\
AGO P4 & AP4 & $82.01^{\circ} \mathrm{S}$ & $96.76^{\circ} \mathrm{E}$ & $80.00^{\circ} \mathrm{S}$ & $41.51^{\circ} \mathrm{E}$ \\
Casey & $\mathrm{CSY}$ & $66.17^{\circ} \mathrm{S}$ & $110.32^{\circ} \mathrm{E}$ & $80.71^{\circ} \mathrm{S}$ & $155.19^{\circ} \mathrm{E}$ \\
Dumont D'Urville & DRV & $66.66^{\circ} \mathrm{S}$ & $140.01^{\circ} \mathrm{E}$ & $80.62^{\circ} \mathrm{S}$ & $235.77^{\circ} \mathrm{E}$ \\
McMurdo & $\mathrm{MCM}$ & $77.85^{\circ} \mathrm{S}$ & $166.70^{\circ} \mathrm{E}$ & $79.94^{\circ} \mathrm{S}$ & $327.53^{\circ} \mathrm{E}$ \\
\hline
\end{tabular}


are smaller than the northern AE (e.g. see Fig. 2), it is important ensure that geomagnetic disturbances are not eliminated when the background level is subtracted at each station.

After subtracting the quiet time average from the $H$ component data, we calculated the $H$ lower (ALS-80) and the $H$ upper (AUS-80) envelopes and their difference $($ AES- $80=$ AUS- $80-$ ALS-80) at one minute resolution, in a manner similar to that used to produce the AE index.

\subsection{Correlations with $\mathrm{Kp}$ and Dst}

AES-80 and AE have been averaged over three hour periods (in UT) for comparison with the Kp index; one hour average values have been considered for comparison with Dst. In Table 2 we show correlation coefficients between these parameters and both the classical electrojet indices and the southern high-latitude indices. The best correlation is found with the $\mathrm{Kp}$ index, indicating that both AE and AES-80 are in good agreement with activity at a planetary level; however the correlation of $\mathrm{Kp}$ with AUS-80 is definitely less significant than with northern AU.

In order to find any possible dependence of the correlations (considered in Table 2) on the UT time, we have calculated correlation coefficients separately for each $2 \mathrm{~h}$ UT time range. In this case hourly averages are considered for indices also in the correlation with $\mathrm{Kp}$ : the same $\mathrm{Kp}$ value has been assigned to each one of the three hours to which it refers. The results are plotted in Fig. 1, where correlation coefficients for AU and AUS80 are shown in the top panels, for AL and ALS-80 in the middle panels and for AE and AES- 80 in the bottom panels. The number of data points in the correlations for each interval is always 122 for northern indices and is between 114 and 117 for southern indices. What can be noted in Fig. 1 is the good agreement between AE and AES-80 independently of UT time (bottom panels). This agreement is a little smaller for the $\mathrm{AL}$ and $\mathrm{AU}$ components. In particular the correlation of $\mathrm{Kp}$ with AUS-80 (top left panel) is significantly lower than with

Table 2. Number of data points $(\mathrm{N})$ and correlation coefficients $(\rho)$ between the indicated parameters

\begin{tabular}{llllll}
\hline & \multicolumn{2}{l}{$\mathrm{K}_{\mathrm{p}}$} & & & \multicolumn{2}{l}{ Dst } \\
\cline { 2 - 3 } \cline { 6 - 6 } & $\mathrm{N}$ & & & $\mathrm{N}$ & $\rho$ \\
\hline AE & 488 & 0.87 & & 1464 & 0.63 \\
$\mathrm{AL}$ & 488 & 0.83 & & 1464 & 0.62 \\
AU & 488 & 0.86 & & 1464 & 0.57 \\
AES-80 & 467 & 0.82 & & 1385 & 0.61 \\
ALS-80 & 467 & 0.76 & & 1384 & 0.54 \\
AUS-80 & 467 & 0.54 & & 1385 & 0.44 \\
\hline
\end{tabular}

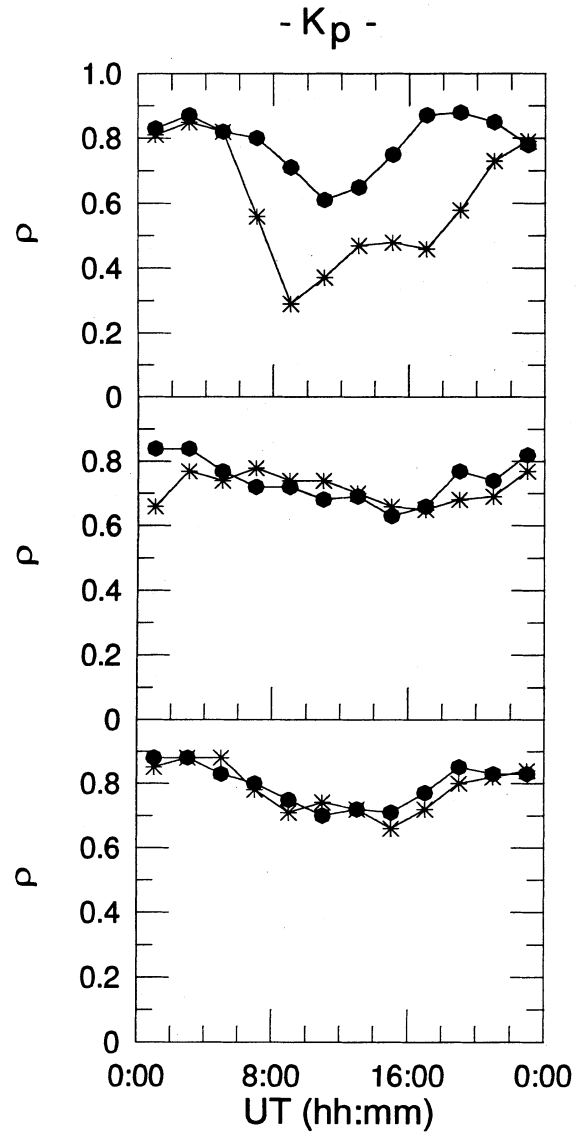

- Dst -

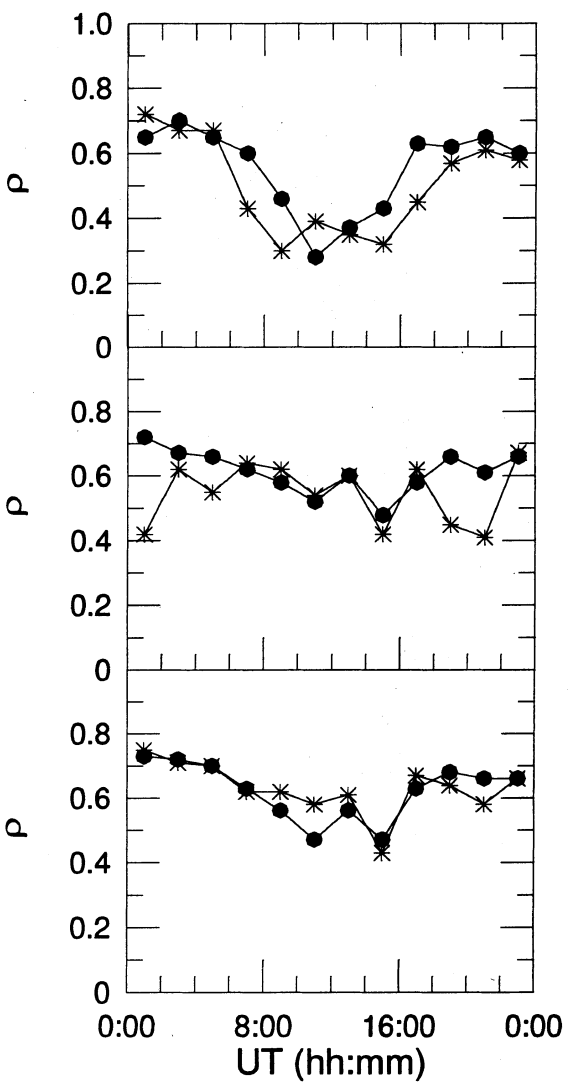

Fig. 1. Correlations of auroral indices and new indices with $\mathrm{Kp}$ and Dst at different UT times. The top panels show correlation coefficients for AU (O) and AUS-80 (*), the middle panels for AL $(\bullet)$ and ALS-80 (*), the bottom panels for AE $(\mathbf{O})$ and AES-80 (*); each point is shown at the centre of the $2 \mathrm{~h}$ interval to which it refers 

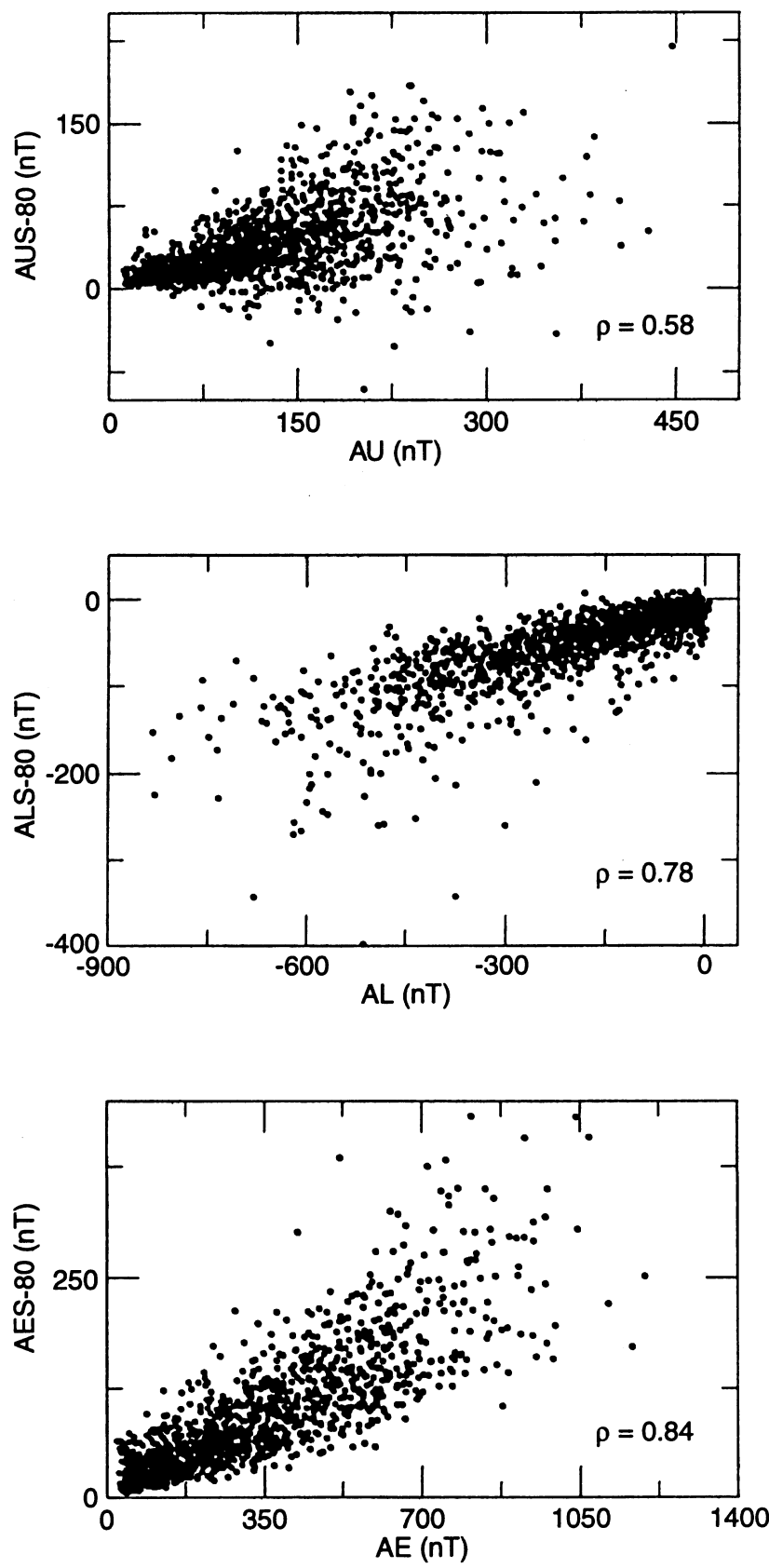

Fig. 2. Scatter plots for AUS-80 and AU (top panel), ALS-80 and AL (middle panel), AES-80 and AE (bottom panel). The number of data points is always $\mathrm{N}=1385$ (hourly values are used in this case) and the correlation coefficient $(\rho)$ is indicated for each panel

$\mathrm{AU}$ for all the time intervals between about $6 \mathrm{UT}$ and about 22 UT.

\subsection{Comparison between southern and northern indices}

In Fig. 2 scatter plots between AUS-80 and AU, ALS80 and AL, AES- 80 and AE are illustrated. A good correlation between AES-80 and AE is shown in the bottom panel; relatively lower correlations are found in the other two cases. In particular a relatively high scatter of data points is found between AUS and AU in the top

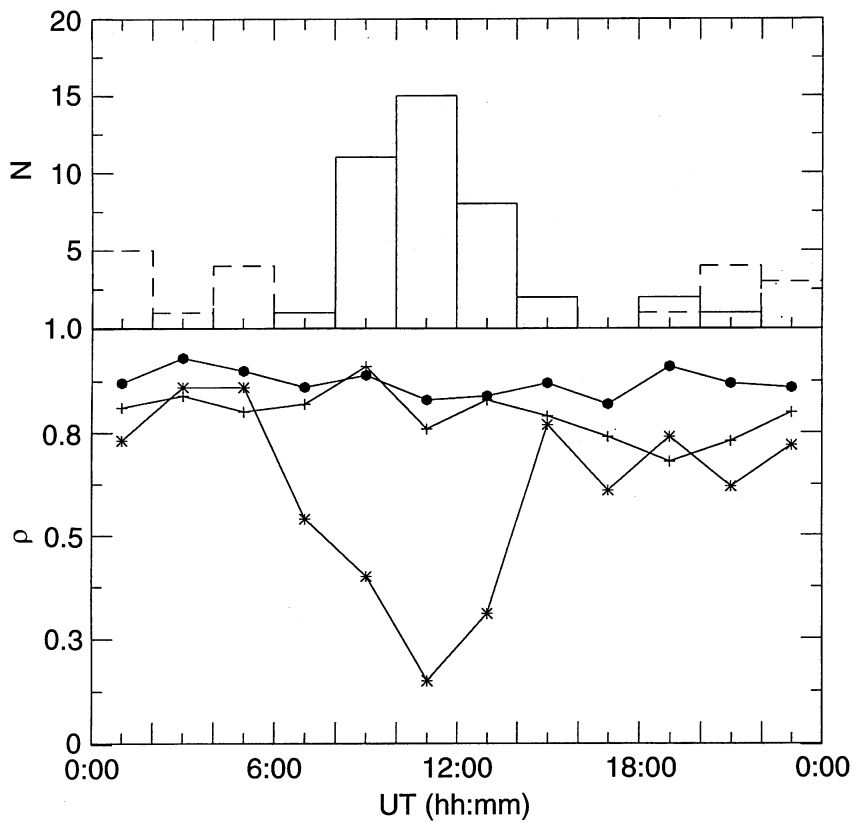

Fig. 3. The upper panel is a histogram of negative AUS- 80 numbers (solid line) and positive ALS-80 numbers (dashed line); the lower panel shows the linear correlations versus UT time (each point is shown at the centre of time interval) between AES-80 and AE (-), ALS-80 and AL (+), AUS-80 and AU (*)

panel. We have considered separately data points corresponding to northward and southward IMF (not shown), but found no significant effects of the IMF- $B z$ component on these correlations.

Correlation coefficients between the northern and southern indices have been calculated separately for each two hour period and the results are illustrated in Fig. 3 (bottom panel), where the upper panel shows the histogram of the number of data points with a negative AUS-80 value (solid line) and with positive ALS-80 (dashed line). A large minimum in the correlations between AUS-80 and AU (*) is found in the time interval 6-14 UT, when a greater number of negative AUS-80 are found. During all of May and June 1994 there were no negative values of one hour average AU. There were five positive values of AL during this period, and 19 positive ALS-80 (distributed over time as illustrated by the dashed line in the top panel of Fig. 3). When considering periods for which $\mathrm{AU}$ is positive while AUS-80 is negative, it is important to recall that AU and AUS- 80 are monitoring two different phenomena: AU is related to the auroral oval eastward currents while AUS- 80 refers to the eastward current component measured in the nominal polar cap region (at about $-80^{\circ}$ ). The presence of negative AU has classically been interpreted as resulting from a uniform field of ring current in the magnetosphere that dominates the zonal current in the local ionosphere (at least above the 12 stations used for deriving AU) (Davis and Sugiura, 1966). However, in the present case, the presence of negative AUS- 80 clustered at a specific UT range does not seem to fit this interpretation. Therefore it is interesting to study possible anomalies in the AUS- 
80 and ALS- 80 relative contributions from each station at different UT time. These contributions are illustrated in Fig. 4, where one minute resolution values have been used for ALS-80 and AUS-80. This figure shows that a greater contribution to AUS-80 and ALS-80, and thereby to AES-80 as well, comes from CSY and DRV than from other stations. This can be simply related to the longitude distribution of stations (see Table 1): AP1, AP4 and MCM are within $80^{\circ}$ of longitude, with the remaining 280 covered only by the two stations CSY and DRV. It appears that each station rather regularly contributes for about half a day to ALS-80 and for the rest to AUS-80, the maximum contribution to AUS-80 being from stations near local geomagnetic midnight (LGM). This is different from the station contribution to the classical auroral electrojet indices and the previous southern AE index calculated from lower latitude stations, where maximum westward currents were detected about 3-4 h after LGM and maximum east-

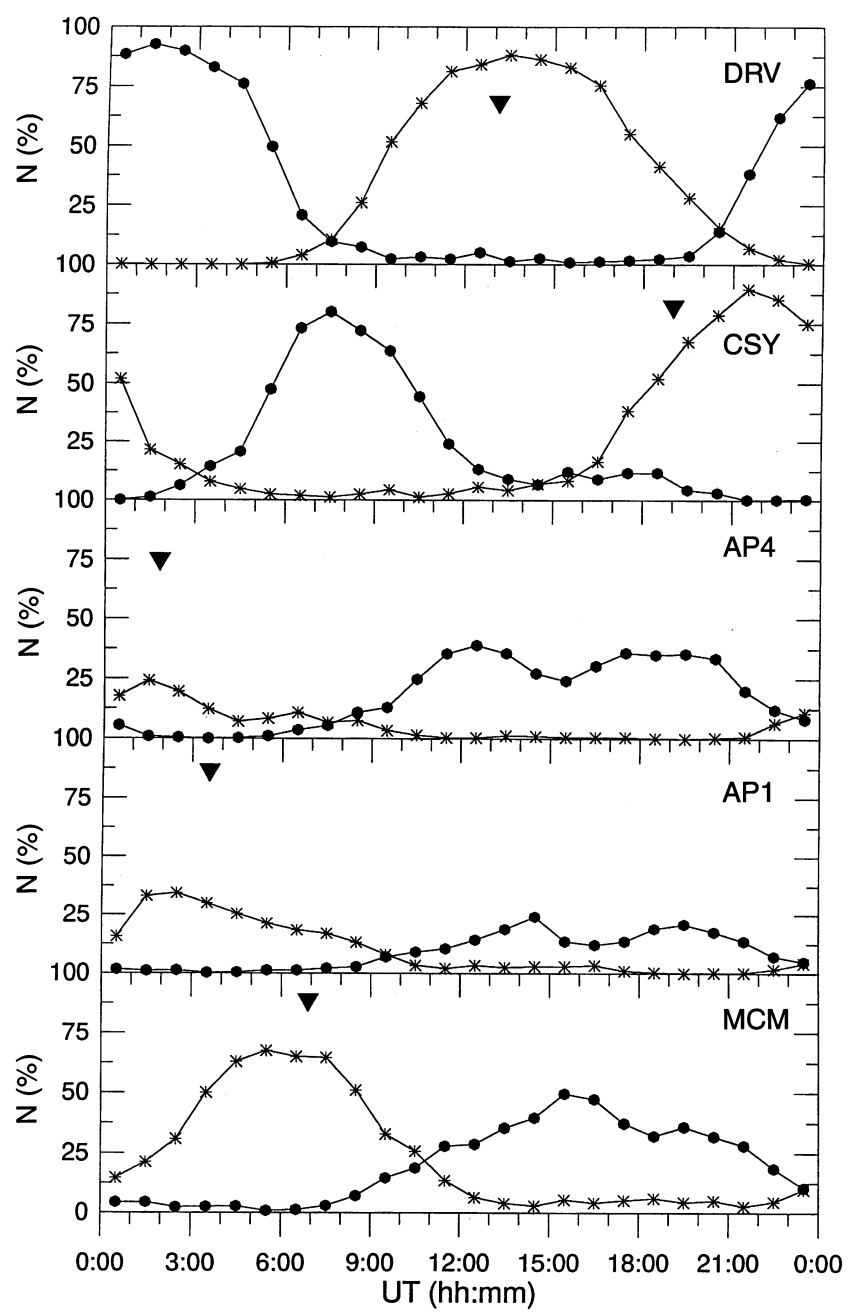

Fig. 4. Percentage of relative contribution from each station to ALS80 (-) and AUS-80 (*) separately for each UT hour. One minute resolution values are considered for ALS-80 and AUS-80. The black triangles indicate the time of local geomagnetic midnight at each station ward currents were detected about 6-7 h before LGM (Allen and Kroehl, 1975; Maclennan et al., 1991).

Looking at Fig. 2 one can see that in general currents detected by AES- 80 are definitely lower than those monitored by AE; this is expected because the average position of the auroral electrojet is at lower latitudes than that of the stations contributing to AES-80. We stress that this level difference observed between AE and AES- 80 is mostly due to the latitudinal difference of the stations and not to a seasonal difference between the two hemispheres. In the Maclennan et al. (1991) paper, where a tentative southern AE index is calculated at the average southern auroral oval latitudes, it is found that the southern AE regression line is very close to the line that corresponds to an equal northern and southern index (in particular it was found: AEsouth $=(0.85 \pm 0.05) \quad$ AEnorth $+65.0 \pm 25.5)$. Although AES-80 is generally lower than AE, it is interesting to see whether there are times when the southern indices measure a perturbation higher than classical indices. To determine this, we calculated the ratios between AES- 80 and AE, ALS- 80 and AL, AUS80 and $\mathrm{AU}$, which represent the percentage of maximum currents detected by classical indices that are revealed by our southern indices. Figure 5 shows the number of data points (indicated as a percentage of the total data points) which are found in each range of values indicated on the abscissa. In most cases we find that the southern polar indices are smaller than the classical indices (i.e. the ratio is less than 1), however there are cases when AES-80 is greater than AE. In the middle panel in Fig. 5, it is interesting to note that in several cases ALS- 80 assumes values more than three times greater than AL.

The average values of $\mathrm{Kp}$ and Dst have been computed separately for each group of data related to each interval on the abscissa of Fig. 5 for the ratios AES-80/AE, ALS-80/AL, and AUS-80/AU. The results are displayed in Fig. 6, where one can see that larger values of AES-80/AE occur for lower $\mathrm{Kp}$ values, i.e. occur in quiet periods when the auroral oval is expected to be more contracted poleward (Kamide et al., 1976). In addition, in Fig. 6 it can be seen that the Dst index attains exceptionally large positive values during the highest AES-80/AE events. This implies that the latter events mainly occur during strong magnetospheric compressions (possibly leading to a storm).

In order to determine the exact position of the auroral oval for the cases when AES- 80 is greater than AE, we have used DMSP satellite data. In particular we present here two cases when the DMSP pass is close to the station contributing ALS- 80 or AUS- 80 value. The indices for the two events are plotted in Fig. 7 and in both cases the station contributing the AUS-80 value that gives the maximum AES-80 is CSY. With respect to the eastward currents measured by AUS-80, it is important to emphasize that the polar projection of the Southern Hemisphere has dusk on the right rather than left and we show in Figs. $8 \mathrm{~b}$ and $9 \mathrm{~b}$ the Northdown view through the Earth. The first event is between 2:00 UT and 4:00 UT on May 1st, the second is between 


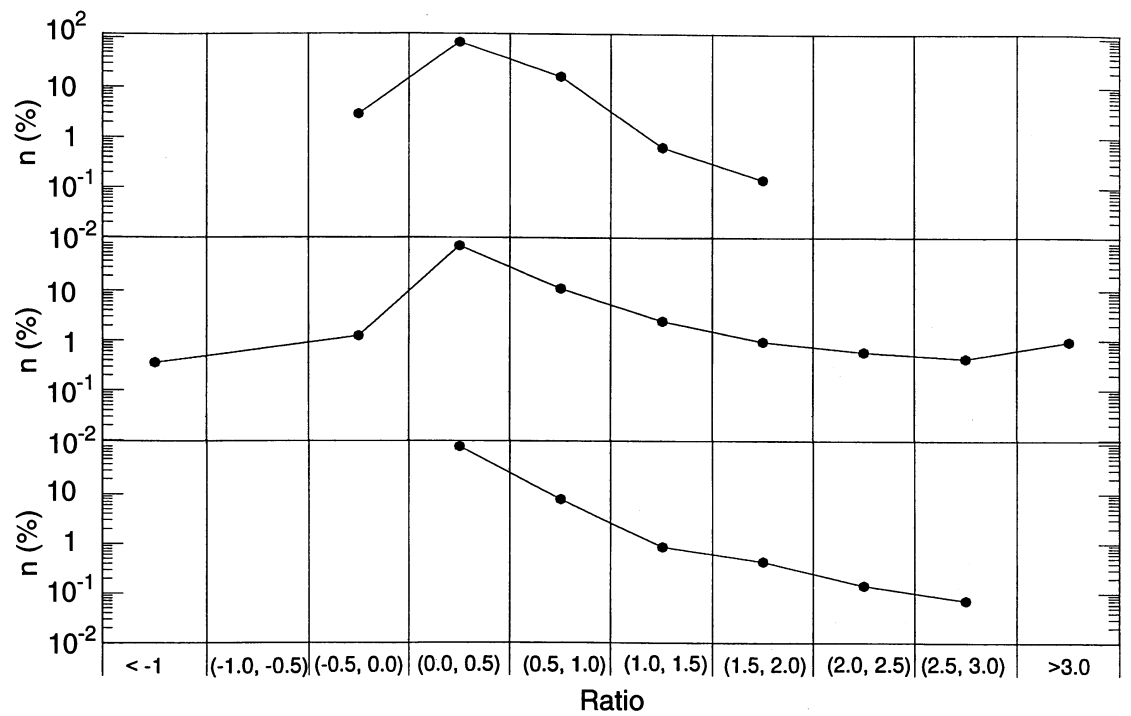

Fig. 5. Percentage of data for which the ratio AUS-80/AU (top panel), ALS-80/AL (middle panel), AES-80/AE (bottom panel) is in the interval indicated on the abscissa. One hour resolution values are used for auroral and new indices in this case

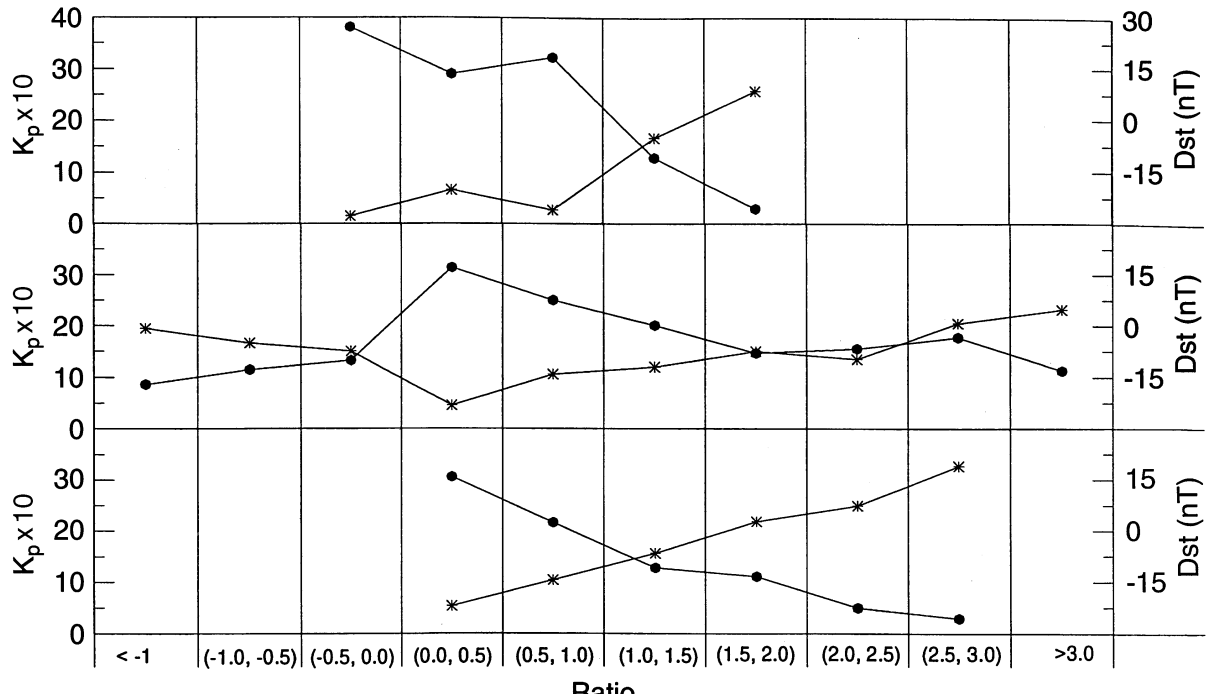

Ratio
Fig. 6. Average value for $\mathrm{Kp}(\mathbf{O})$ and Dst (*) for the groups of data binned into the same AUS-80/AU (top panel), ALS-80/AL (middle panel) and AES-80/ $\mathrm{AE}$ (bottom panel) ratio ranges as in Fig. 5
1:00 UT and 2:00 UT on May 23rd. For these periods the hourly average values of AE, AES-80, Kp and Dst are reported in Table 3.

Figure 8a shows the DMSP pass for the event on May 1st: the auroral oval boundaries are annotated with arrows and correspond, respectively from dawn to dusk, to $69.9^{\circ} \mathrm{S}, 83.5^{\circ} \mathrm{S}, 83.3^{\circ} \mathrm{S}$ and $71.2^{\circ} \mathrm{S}$ corrected geomagnetic latitudes. The position of CSY with respect to the DMSP pass (shown by the asterisk in Fig. 8b) is rather close, and the location of CSY seems to be well within

Table 3. Average values for AE, AES-80, Kp and Dst during the indicated one hour intervals

\begin{tabular}{llll}
\hline & $\begin{array}{l}\text { May 1st } \\
(2.0-3.0) \mathrm{UT}\end{array}$ & $\begin{array}{l}\text { May 1st } \\
(3.0-4.0) \mathrm{UT}\end{array}$ & $\begin{array}{l}\text { May 23rd } \\
(1.0-2.0) \mathrm{UT}\end{array}$ \\
\hline $\mathrm{AE}(\mathrm{nT})$ & 23 & 31 & 48 \\
$\mathrm{AES}-80(\mathrm{nT})$ & 66 & 53 & 65 \\
$\mathrm{~K}_{\mathrm{p}} \times 10$ & 3 & 17 & 10 \\
Dst (nT) & 19 & 19 & -11 \\
\hline
\end{tabular}

the auroral oval precipitation region. For the second selected event (Fig. 9) we obtained a DMSP pass exactly over the CSY location, which is again well within the auroral oval (arrows indicating boundaries in Fig. 9a are respectively from dawn to dusk at: $70.0^{\circ} \mathrm{S}, 80.0^{\circ} \mathrm{S}$, $69.9^{\circ} \mathrm{S}$ and $67.2^{\circ} \mathrm{S}$ ). In this second case we see that there is a portion of the auroral oval below latitude $70^{\circ} \mathrm{S}$, however it is rather narrow (about $2^{\circ}$ of latitude from the poleward $69.9^{\circ} \mathrm{S}$ boundary) and it does not cover most of the standard AE latitude range.

\section{Discussion and conclusions}

In order to test the quality of AES-80 as a global average activity level indicator, its correlations with $\mathrm{Kp}$ and Dst (illustrated in Fig. 1) can be considered. These correlations are very similar to those obtained for the AE index at all UT times, indicating a good relationship between the two indices. The main differences are related to the AUS- 80 parameter: in the time range 

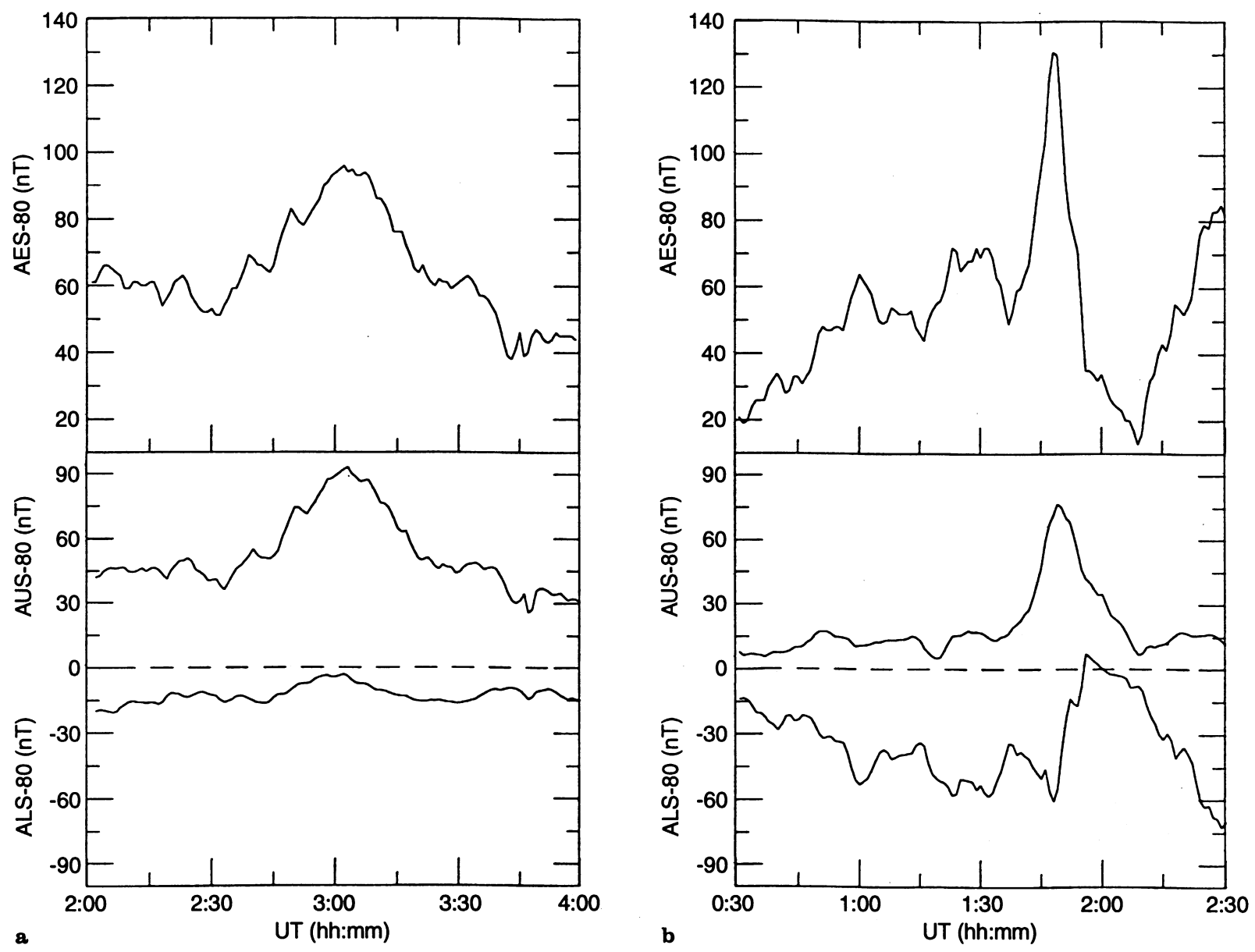

Fig. 7a,b. Plot of AES-80, ALS-80 and AUS-80 for two specific periods with high activity in AES-80 on a May 1st and b May 23rd 1994

about 6-22 UT, AUS-80 shows features that differ from $\mathrm{AU}$ with respect to the correlations with $\mathrm{Kp}$. This may be related to the seasonal dependence of AU reported by Allan and Kroehl (1975); or it could be related to the lower correlations between AU and AUS-80 occurring in about the same UT time range (illustrated in Fig. 3). Both results could be due to the increasing number of negative AUS- 80 values clustered around 12 UT, which could suggest that AUS-80 does not monitor eastward currents well at this time, possibly due to the distribution of contributing stations. However this seems unlikely as the total number of negative AUS- 80 values is less than $1 \%$ of the total number of data points. In addition we emphasize that, for correlations between the absolute values of AU and AUS-80, the correlation coefficients are significantly higher in the specific time range 6-14 UT. This seems to suggest that the cluster of negative AUS- 80 around time $10-12$ UT is accidental (due to the specific two month period considered) and does not imply a smaller significance for AUS-80. It is worthwhile to clarify that a negative AUS- 80 value can be interpreted to mean that the ionospheric eastward current is not as important as further magnetospheric effect on the measured parameter AUS-80. This interpretation is similar to the interpretation of negative $\mathrm{AU}$ given by Davis and Sugiura (1966) for auroral latitudes.
Thus in particular negative AUS-80 means that eastward current intensity is so low that it cannot be detected by the index.

In previous terms, we can say that the differences observed between the northern AU and the southern AUS- 80 can be easily related to the seasonal dependence of eastward currents and to the fact that AUS- 80 refers to the average polar cap location while AU refers to the eastward auroral electrojets. In fact certainly we expect that eastward currents at $-80^{\circ}$ are less intense than the eastward electrojets at the auroral oval average location. In addition, due to the seasonal effect, we expect in general less intense eastward currents in the Southern Hemisphere than in the Northern. In particular it is known that westward currents are less influenced by seasonal effects than are the eastward currents (e.g. Allen and Kroehl, 1975). This could explain why differences between AU and AUS-80 are more important than between AL and ALS-80 in our results.

Maclennan et al. (1991), who calculated a southern AE index using stations from nominal auroral oval average latitudes (which are clustered in longitude in the southern hemisphere), found an increased correlation between AE and AES in the time range between 00:00 and 11:00 UT, indicating that the quality of southern $\mathrm{AE}$ was better at these times. In our case no significant 


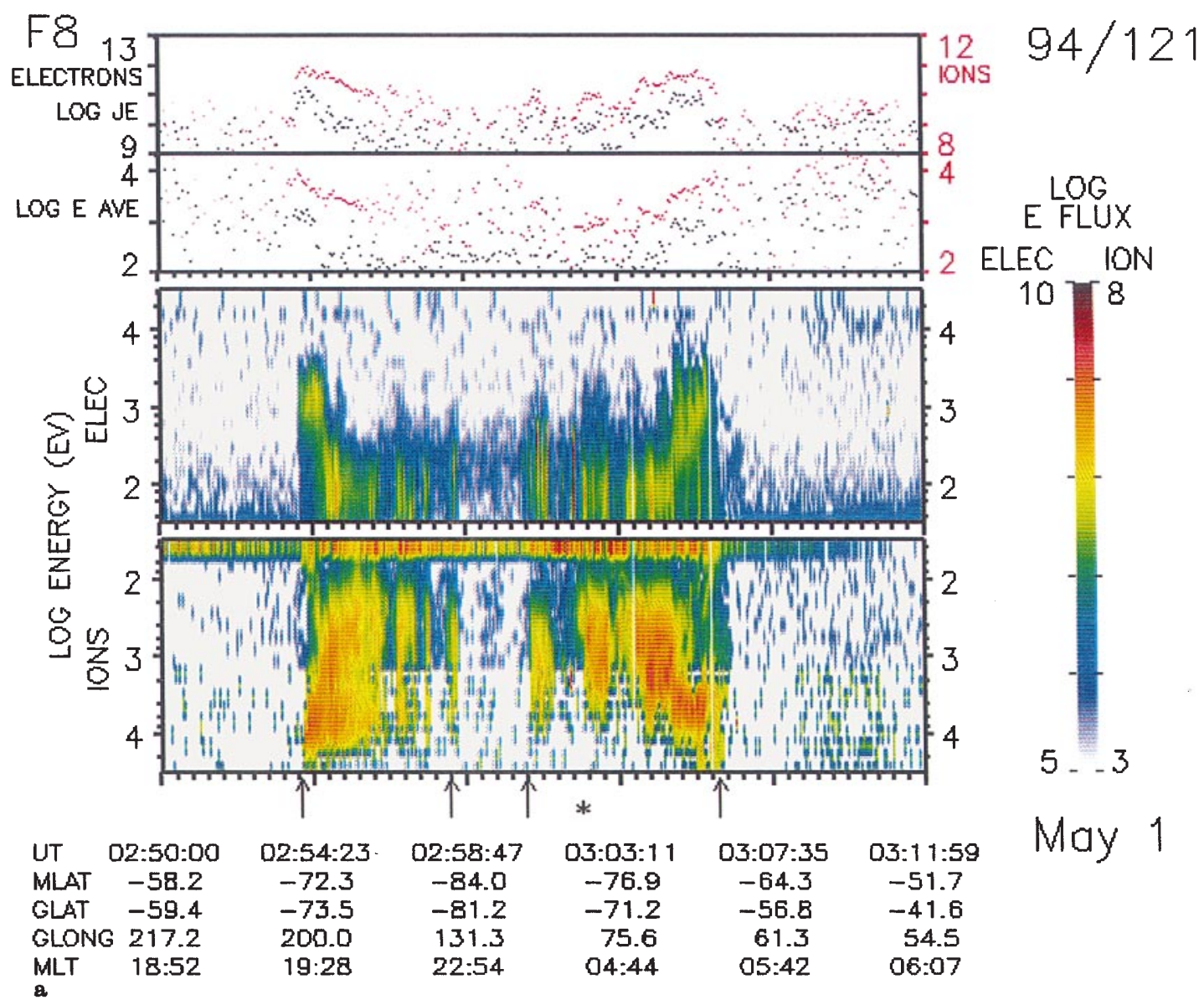

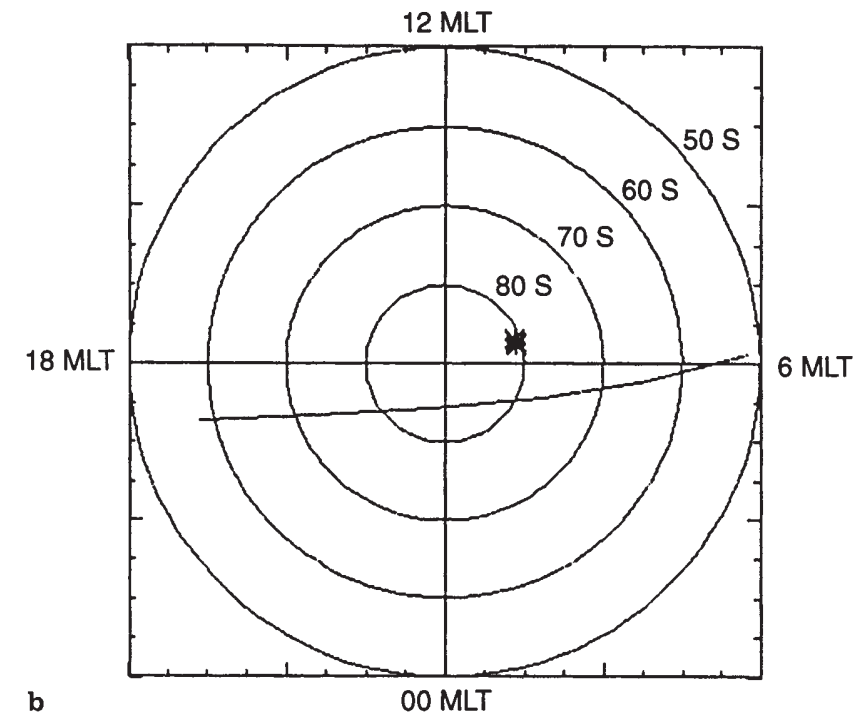

time dependence of the AE/AES- 80 correlation is found. This could be simply due to the different latitudes of the stations considered. AES- 80 is not designed to monitor the maximum electrojet current (as $\mathrm{AE}$ ), but it is correlated with this maximum and it can be the maximum itself (even attaining values greater than AE) when the auroral oval is sufficiently contracted poleward. Figure 2 shows that in general AES-80 assumes values smaller than AE. The different nature of AES- 80 with respect to the southern AE calculated by
Fig. 8. a Spectrogram of particle precipitation measured by DMSP-F8 satellite at the same UT time as the event displayed in Fig. 7a. The arrows indicate the auroral oval boundaries and the asterisk the position closest to CSY station. b Pass of DMSP-F8 satellite with the position of CSY (indicated by *) during UT time displayed in the spectrogram

Maclennan et al. (1991) can explain our result of finding a significant correlation between AE and AES-80 at all universal times.

The present station distribution for AES-80 (Table 1) can easily explain the greater relative contribution of CSY and DRV to the ALS-80 and AUS-80 values shown in Fig. 4. The main characteristic that emerges from this figure is that the maximum AUS- 80 contribution comes from times around local midnight and the maximum ALS- 80 contribution is almost half a day 


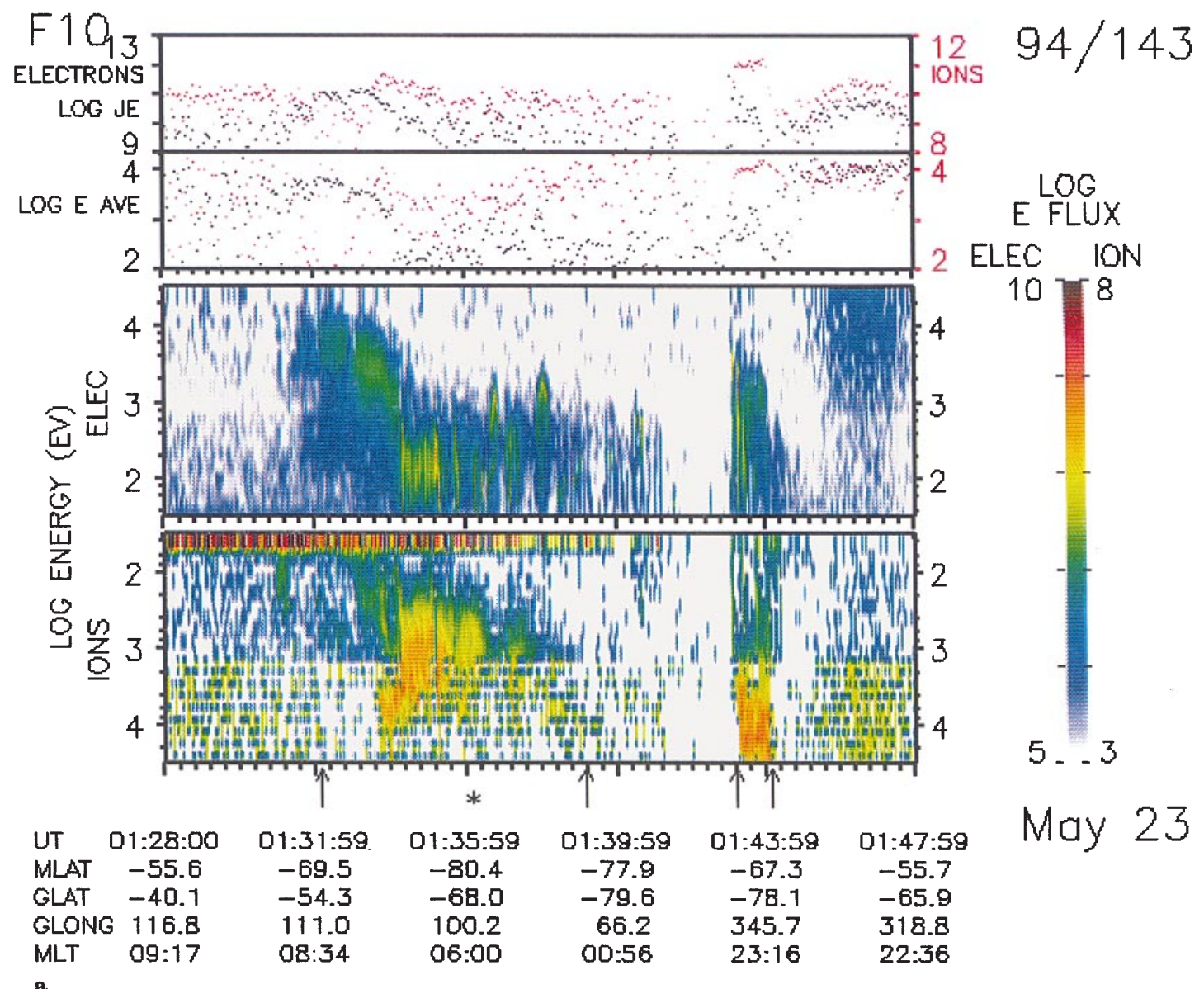

a

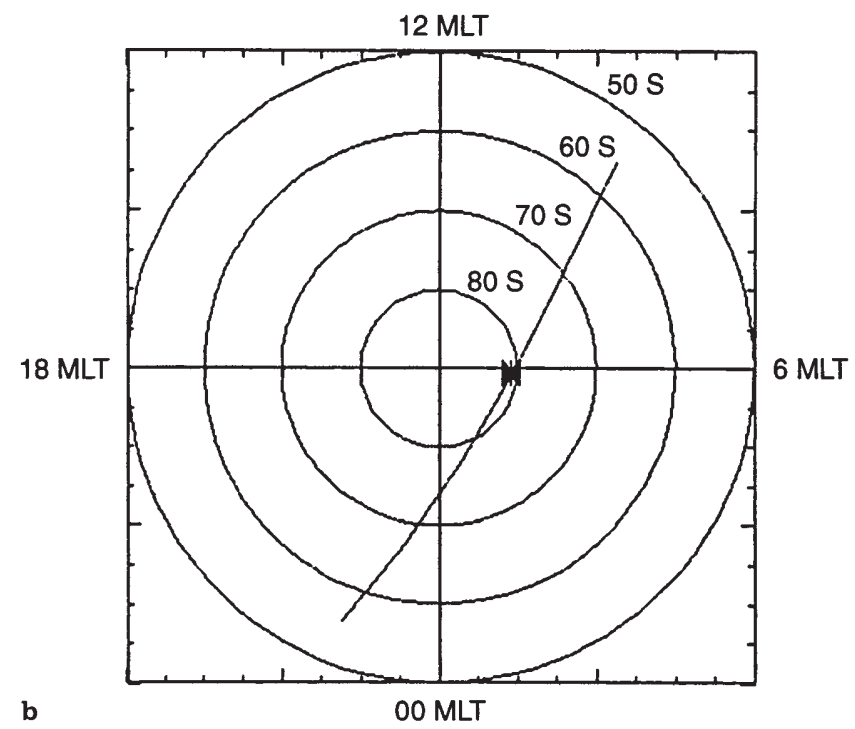

Fig. 9. a Spectrogram of particle precipitation measured by DMSP-F8 satellite at the same UT time as the event displayed in Fig. 7b. The arrows indicate the auroral oval boundaries and the asterisk the position closest to CSY station. b Pass of DMSP-F8 satellite with the position of CSY (indicated by *) during UT time displayed in the spectrogram

away from this. As mentioned, this result is completely different from previous findings at auroral oval latitudes (Allen and Kroehl, 1975; Maclennan et al., 1991) and is, as expected, clearly inconsistent with the ionospheric current pattern associated with the auroral latitude substorms.

However this does not mean that weak polar cap substorms cannot be detected by AES- 80 . Such sub- storms are not likely to be detected by PC because that index is derived from a single nearpole station; as AES80 uses information from several longitudinally distributed sources, detection of localized high-latitude substorms is more probable.

Although in general AES-80 is smaller than AE, we find several cases where larger electrojets are indicated by AES- 80 than AE. 
The number of events with AES-80/AE greater of 1 is not null; in fact we find that the ratio ALS-80/AL reaches values greater than 3 , indicating that the maximum westward electrojet can be monitored at $80^{\circ} \mathrm{S}$. The occurrence of these maximum electrojets at what are considered to be polar latitudes is associated with low values of $\mathrm{Kp}$ (smaller than 2) and with Dst near zero or positive; this is in agreement with the poleward shift of the auroral oval during the quietest periods (Kamide et al., 1976). We have found evidence of the exact position of the auroral oval in two specific cases with AES-80 $>$ AE and we have seen that in effect all or almost all the oval was at latitudes greater than $70^{\circ} \mathrm{S}$ at these times in the Southern Hemisphere.

We conclude that AES- 80 can be larger than AE when the auroral oval is contracted and reaches $-80^{\circ}$ of latitude. Moreover, because of its good correlation with AE at all times, AES- 80 could be useful as an indicator of global electrojet activity in the Southern Hemisphere, where sufficient ground-based information is not available at nominal auroral oval latitudes. As with all indices, several contributing sources are present for AES-80, and further studies are being performed to identify whether specific AES-80 enhancements are due to polar cap currents, to auroral oval movement towards the cap, or to auroral electrojets and the occurrence of polar cap substorms.

Acknowledgements. Thanks are due to L.J. Lanzerotti (Bell Laboratories, Lucent Technologies, Murray Hill, NJ) for helpful comments and suggestions. The authors thank T.Kamei from WDC-C2 in Kyoto for providing the official auroral indices data. MJE acknowledges support from NSF grant OPP-9529177 to the University of Maryland, and by subcontract to Augsburg College. The U.S. investigators acknowledge the support of the Office of Polar Programs, National Science Foundation, for upper atmosphere physics programs at McMurdo and the AGOs. This research was supported in part by the Italian Antarctic Research Program (PNRA).

Topical Editor D. Alcaydé thanks K. Mursula and R. L. McPherron for their help in evaluating this paper.

\section{References}

Allen, J. H., and H. W. Kroehl, Spatial and temporal distributions of magnetic effects of auroral electrojets as derived from AE indices, J. Geophys. Res., 80, 3667, 1975.

Ballatore, P., C. G. Maclennan, and M. Candidi, Some aspects of geomagnetic disturbances at polar latitudes, Conf. Proc., 58, 97-100, Ed. S.I.F., Bologna, 1997.

Baumjohann, W., Merits and limitations of the use of geomagnetic indices in solar-wind magnetosphere coupling studies, in SolarWind Magnetosphere Coupling, Ed. Y. Kamide and J. A. Slavin, Terra Scientific, Tokyo, 1986.

Belon, A. E., J. E. Maggs, T. N. Davis, K. B. Mather, N. W. Glass, and G. F. Hughes, Conjugacy of visual auroras during magnetically quiet periods, J. Geophys. Res., 74, 1, 1969.

Davis, T. N., and M. Sugiura, Auroral electrojet activity index AE and its universal time variations, J. Geophys. Res., 71, 785, 1966.

Engebretson, M. J., and the PENGUIn/AGO team, The United States Automatic Geophysical Observatory (AGO) program in Antarctica, in Satellite-Ground Based Coordination Sourcebook, Ed. M. Lockwood et al., European Space Agency Special Publication, SP-1198, pp.65-99,1997.

Kamide, Y., J. L. Burch, J. D. Winningham, and S. I. Akasofu, Dependence of the latitude of the cleft on the interplanetary magnetic field and substorms activity, J. Geophys. Res., 81, 698, 1976.

Maclennan, C. G., L. J. Lanzerotti, S.-I. Akasofu, A. N. Zaitzev, P. J. Wilkinson, A. Wolfe, and V. Popov, Comparison of electrojet indices from the northern and southern hemispheres, $J$. Geophys. Res., 96, 267, 1991.

Rycroft, M. J., Antarctic observations available for IMS correlative analyses, in The IMS Source Book, Eds. C. T. Russell, and D. J. Southwood, American Geophysical Union, pp 196-210, 1982.

Troshichev, O. A., V. G. Andrezen, S. Vennerstrøm, E. FriisChristensen, Magnetic activity in the polar cap - a new index, Planet. Space Sci., 36, 1988.

Vennerstrøm, S., E. Friis-Christensen, O. A. Troshichev, and V. G. Andrezen, Comparison between the polar cap index, pc, and auroral electrojet indices AE, AL, and AU, J. Geophys. Res., 96, $101,1991$.

WDC-C2 for Geomagnetism, Date Book 22, Faculty of Science of Kyoto University (Kyoto) and National Institute of Polar Research (Tokyo), 1993

Weatherwax, A. T., T. J. Rosenberg, C. G. Maclennan, and J. H. Doolittle, Substorm precipitation in the polar cap and associated Pc5 modulation, Geophys. Res. Lett., 24, 579, 1997. 